BMJ Nutrition,

Prevention \& Health

\section{Effects of SARS-CoV-2 pandemic on follow-up and pharmacological treatment of chronic diseases in undocumented migrants}

To cite: Fiorini G, Franchi M, Corrao G, et al. Effects of SARS-CoV-2 pandemic on follow-up and pharmacological treatment of chronic diseases in undocumented migrants. BMJ Nutrition, Prevention \& Health 2021:4:e00274. doi:10.1136/ bmjnph-2021-000274

For numbered affiliations see end of article.

Correspondence to Dr Silvano Gabriele Cella, Clinical Sciences and Community Health, University of Milan, 20129 Milano, Italy; silvano.cella@unimi.it

Received 22 March 2021 Accepted 28 May 2021 Published Online First 2 July 2021
Check for updates

(c) Author(s) (or their employer(s)) 2021. Re-use permitted under CC BY-NC. No commercial re-use. See rights and permissions. Published by BMJ.

\section{ABSTRACT}

Background All over the world, the COVID-19 pandemic, not unlikely other epidemics, has hit harder people in low socioeconomic conditions.

In Western countries, undocumented migrants are a growing component of this disadvantaged segment of the population. Their health conditions are frequently burdened by a number of chronic conditions, and they experience many difficulties in accessing public health services. Frequently, the only medical assistance they can get is provided by nongovernmental organisations.

Methods We studied the medical records (including pharmacological treatments) of all patients attending the outpatient clinics of Opera San Francesco (OSF; a big charity in Milano, Italy), in the first 5 months of 2020 . These comprise the outbreak of the pandemic and the lockdown period. The 1914 patients (1814 undocumented migrants and 100 Italians) seen during the lockdown were compared with those seen in the same period of 2019 and with those seen in the preceding months of 2020. We especially focused on three chronic conditions: cardiovascular diseases, diabetes and psychiatric disorders.

Results The number of consultations during the first 5 months of 2020 was much smaller than that of the same period of 2019. During the lockdown, we found 4048 consultations for 1914 patients, while they were 8051 in the same period of 2019 and 5681 in the first 2 months of 2020 . The quantity of medicines dispensed by OSF showed a marked decrease in the period of the study and mainly during the lockdown.

The decrease in consultations and dispensation of medicines was most evident for psychiatric patients and almost not existent for patients with diabetes. Female patients suffered a more pronounced reduction.

Conclusions Western countries need strategies to better assist the very poor during epidemics.

Differences among different groups of disadvantaged persons should be taken into account when designing recovery plans.

\section{INTRODUCTION}

The SARS-CoV-2 emerged in late 2019. By the beginning of 2020, it became pandemic, and containment measures began to be
Key messages

What is already known?

- Undocumented migrants are a growing and disadvantaged part of the population in many countries all over the world.

- Beside other difficulties, they have many problems in accessing even limited forms of medical assistance.

- Epidemics hit more severely persons who live in very poor socioeconomic conditions.

- Very little is known about the health conditions of undocumented migrants during COVID-19 pandemic.

What are the new findings?

- Undocumented migrants attending the outpatient clinics of a big charity in Milan, Italy, were much fewer than usual during the first wave of the pandemic and the following lockdown.

- This reduction in visits to the clinic affected also patients with chronic conditions, especially those with mental disorders, but not those with diabetes.

- Females suffered a greater reduction than males.

What do the new findings imply?

- Undocumented migrants with different chronic conditions behave differently. This can be important when evaluating their awareness of disease and long-term adherence to treatment.

- Strategies should be implemented to ensure medical assistance to undocumented migrants and especially to those among them who appear to be more vulnerable: patients with mental health problems and female patients.

- This should be considered both in periods of emergency, as COVID-19 pandemic, and when designing truly equitable recovery plans.

implemented all over the world. In Italy, the first diagnosis of SARS-CoV-2 infection was made on 31 January 2020, when two Chinese tourists tested positive, but the virus is thought to have been present since many weeks before. 
The government decided containment measures for almost all Northern Italy on 8 March 2020. These imposed severe restrictions on all non-indispensable activities and displacements. This lockdown continued until the end of May, when containment was progressively lifted. Respecting social distancing, wearing a face mask and avoiding excessive gathering together have never been cancelled, in consideration of the continuing presence of SARS-CoV-2 and its known mechanism of transmission. ${ }^{12}$

It has been demonstrated that containment measures can be at least partially effective in slowing down the spread of the virus and the consequent increase in mortality, though their efficacy depends on the level of virus penetration at the onset of confinement. ${ }^{3-5}$

Besides their impact on the epidemiology of COVID19 , these measures had multifaceted consequences both on people's health and well-being.

Psychological effects have been described by many researchers; they include emotional disturbances as anxiety, anger, confusion, depression and somatic symptoms as palpitations, breathing difficulties and insomnia. ${ }^{6}$ These psychological disturbances appeared to be dependent both on non-modifiable (eg, female gender and work uncertainty) and modifiable (eg, physical exercise and prayer) factors; thus, the degree of resilience varied among individuals of the same population. ${ }^{78}$ Some authors have also suggested that adolescents during COVID-19 lockdown were at risk for post-traumatic stress disorder and depressive and anxiety disorders; moreover, those already affected by psychiatric problems could experience a worsening of their symptoms. ${ }^{9}$ Therefore, younger age and pre-existing psychiatric disturbances could be other important factors regulating the psychological impact of lockdown.

Both the pandemic itself and the lockdown had considerable effects on a number of diseases, though the role of confounding factors should also be considered. Age, for example, has an effect on the consequences of quarantine, ${ }^{79}$ but it also has a role in determining susceptibility to infection. ${ }^{10}$ This has an important consequence: populations of different age would have a different COVID-19 epidemiology. ${ }^{11}$ For example, in countries with younger populations, as low-income countries, the expected incidence of COVID-19 and the consequent case fatality rates would be lower. ${ }^{12}$ However, this could be at least in part counterbalanced by the shortage of adequate health resources, as it is unfortunately often the case in these countries. Both in low-income and high-income countries comorbidities and pre-existing non-communicable diseases (NCDs) play also a role. ${ }^{13}$ In the former, it is possible that during a pandemic they could obtain even less attention than that they usually receive. ${ }^{14}$ In the latter they could be associated with a worse outcome, since they are more frequent in the elderly. However, pre-existing NCDs are not only associated with a worse outcome of SARS-CoV-2 infection. ${ }^{13}$ These diseases can be affected by the lack of physical exercise, the increased frequency of unhealthy lifestyles and the reduction of nutrients as vitamin D. ${ }^{15}$

Moreover, NCDs usually need continuous tratment, frequent adjustments of pharmacological therapies, periodic check-up and laboratory tests and, often, daily care by health professionals. All this becomes difficult during a pandemic and especially during a period of quarantine. ${ }^{15}$ During the lockdown, nearly all patients with chronic conditions experienced changes in their everyday life, and almost one half of them reported an impact on their health conditions. ${ }^{16}$

Another important aspect is the reduction in hospital admissions for acute conditions, an example being acute coronary syndrome; this trend has been observed worldwide, even in countries with a low incidence of COVID$19 .{ }^{17}$ The explanation is not yet conclusive. Fear to access emergency departments crowded with patients with COVID-19 may have a role, but the incidence of myocardial infarction immediately after lockdown could be truly reduced, as a consequence of reduced exposure to triggers as air pollution. ${ }^{18}$

Another very important consideration has to be made: epidemics, and the present is no exception, are not socially neutral. Since the 1918 Spanish influenza epidemic, we know that their incidence is different in persons of different economic conditions, with disadvantaged people being more affected. ${ }^{19}$ This difference in morbidity and mortality reflects pre-existing ethnic and socioeconomic differences together with differences in chronic diseases and the social determinants of health. ${ }^{20}$

Moreover, lockdown implementation worsens the conditions of vulnerable parts of the population, ${ }^{21}$ as undocumented migrants. ${ }^{22}$

More in general, it is now clear that in Western countries the most vulnerable segments of the population were hit more severely, and undocumented migrants are probably in the first place. ${ }^{23}$

This study evaluates a large sample of undocumented migrants attending a charitable outpatient clinic in Milano, Italy, in the months from the outbreak of the pandemic to the end of lockdown. Our aim was to evaluate the response to their health needs. We mainly focused on the necessity, for patients with chronic conditions, to continue their therapy without interruptions. This knowledge could be valuable to plan interventions for these and other disadvantaged persons during epidemics.

\section{METHODS \\ Population}

We studied the medical records of all the persons attending the outpatient clinics of Opera San Francesco $(\mathrm{OSF})$, a non-governmental organisation (NGO) in Milan, Italy, assisting the poor. The population relying on OSF for medical assistance is predominantly composed of undocumented migrants, with a minority of indigent Italians. OSF has an electronic database of all patients. For each of them, demographic data, consultations, tests and 
dispensations of medicines are recorded. Doctors run the clinics on a voluntary basis. Medicines are given for free to the patient at the end of each consultation, according to doctor's prescription. The pharmacists keep a digital record of each dispensation, which includes the drug identified according to the Anatomical Chemical Therapeutic (ATC) classification and the amount dispensed according to the Defined Daily Dose (DDD). ${ }^{24}$ We have previously described this system of charitable health assistance in greater detail elsewhere. ${ }^{25}$

In this study, we evaluated the medical records of the patients who received medical assistance at OSF in the first 5 months (7 January to 31 May) of 2019 and 2020. These are the months comprising, in 2020, the prodromal phase, the outbreak and the attempt of containment (lockdown) of the pandemic.

After this preliminary analysis, we compared the data of the patients attending the charity from 8 March 2020 to 31 May 2020 (ie, the strict lockdown period; N=1914), with those of two other groups of patients. The first was made of all the patients who had received medical assistance by OSF in the same period of the previous year $(\mathrm{N}=3403)$; the second comprised all the patients seen at OSF from 7 January 2020 to 3 March 2020 (prelockdown period; $\mathrm{N}=2596$ ). We considered necessary this double comparison, since this population is very unstable.

\section{Statistical analysis}

We produced descriptive tables showing the distribution of the number of drug prescriptions, overall and stratified by ATC classes of drugs used in the treatment of common NCD, in the lockdown period of 2020 as compared with the same period of 2019 and the prelockdown period of 2020. We considered the following NCD: cardiovascular diseases (ATC class C), diabetes (ATC class A10) and psychiatric disorders (ATC classes N05-N07). We then calculated the per cent changes in the number of prescriptions in the lockdown period, as compared with those prescribed in the same period of 2019 and in the prelockdown period of 2020.

We graphically represented the weekly distribution, along with $95 \%$ CIs, of the number of both drug prescriptions and consultations in the three considered periods by using the local polynomial regression smoothing (LOESS) method. ${ }^{26}$

In addition to the trend of the number of drug prescriptions, we also evaluated the temporal trend of the weekly distribution of drug quantity (expressed in terms of DDD/1000 patients), overall and stratified by the aforementioned specific ATC classes, in each of the three periods considered.

We further stratified the previous analyses, where appropriate, by age, sex and ethnicity.

All analyses were conducted using the V.9.4 of the SAS package (SAS Institute).

\section{Patient and public involvement}

This study does not involve patients and public consultation since it was based on electronic records available to

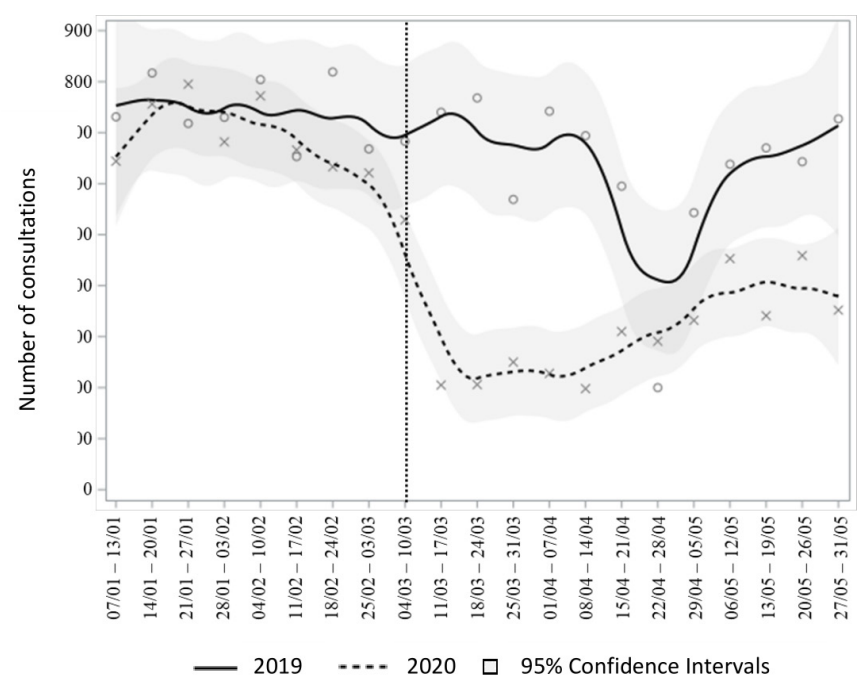

Figure 1 The figure shows the trend of the number of consultations at OSF during the period from 7 January 2019 to 31 May 2019 (solid line) and the same period in 2020 (dotted line). The vertical line indicates the date the lockdown was imposed in Italy (ie,8 March 2020). Trends of the number of consultations were smoothed using the LOESS method, in order to remove seasonality and casual fluctuations over time. Circles and crosses represent the number of visits observed in 2019 and in 2020, respectively. The grey areas represent $95 \%$ Cls. OSF.

researchers on demand, after approval of the appropriate ethics committee.

\section{RESULTS}

At a preliminary analysis, we noticed that the number of consultations at OSF was much lower in the first 5 months of 2020 (from 7 January to 31 May) than in the corresponding period of 2019. This decrease was more evident in the weeks from 8 March to 31 May, corresponding to the period of lockdown (figure 1). Concomitantly, we observed a reduced dispensation of medications in the first 5 months of 2020 (26 292 in 2020 vs 41057 in 2019, $-36.0 \%)$.

The decrease in consultations during the lockdown was paralleled by a decrease in the number of patients accessing OSF outpatient clinics (1914 in 2020 vs 3403 in 2019).

The 1914 patients attending OSF during the lockdown were 914 males and 1000 females. Their median age was 42 years $(q 1=31, q 3=53)$. The majority $(n=1740 ; 91.1 \%)$ was in the $18-64$ years age range; 44 patients $(2.3 \%)$ were under 18 years, and 127 (6.6\%) were 65 years and over. For two patients, age was missing.

They came from the following geographical macro areas: Mediterranean Africa: 253 (13.2\%), sub-Saharan Africa: 116 (6.1\%), Latin America: 909 (47.5\%), Asia: 198 (10.3\%), Eastern Europe: 324 (17.0\%) and other geographical areas: $112(5.8 \%)$. The latter was composed almost entirely of Italians $(\mathrm{n}=100)$. Information on geographical provenance was missing for two patients. 
The results of the comparison with patients seeking medical assistance in the same period of 2019 and with those seen from 7 January 2020 to the beginning of lockdown are presented further.

\section{Lockdown versus same period of $\mathbf{2 0 1 9}$}

Though the reduction in the number of consultations during the lockdown (4154 vs 8212) was generalised, we focused on patients with three frequent chronic conditions: diabetes, cardiovascular diseases and psychiatric disorders. We evaluated the quantity and number of dispensations of medicines used in the treatment of these conditions. As specified previously, drugs were identified with their ATC code.

As shown in figure 2, we found that the number of dispensations was lower in 2020 for cardiovascular and psychiatric diseases, especially during the lockdown period (right part of the plots); on the contrary, dispensation of antidiabetic agents remained substantially unchanged. The dispensed quantity, measured as DDD/1000 patients, was also lower for cardiovascular and psychiatric diseases in 2020, especially during lockdown; again, this was not the case for diabetes (figure 3 ).

Splitting antidiabetic medications into insulins and oral antidiabetics, we noticed that a reduction in the dispensation of the latter $(-18.9 \%)$ was compensated by an increased demand for the former $(+26.4 \%)$.

All the changes described for the lockdown period, as compared with the same period of 2019, affected all ethnicities, with the exception of the small number of indigent Italians who had a reduction only of psychiatric patients seen at OSF. All age groups showed comparable trends, while females experienced a greater reduction in the number of patients in each NCD group (table 1).

\section{Lockdown versus prelockdown period of 2020}

These two periods had a different duration: 3 months for lockdown and 2 months for prelockdown. In spite of this, less patients were seen (1914 vs 2596) and less consultations were carried out (4154 vs 5569) during the lockdown. Again, we considered the medications used to treat the more common NCD. The weekly pattern for number of prescriptions and dispensed DDD/1000 patients is shown in figures 2 and 3 (dotted lines). A decrease during lockdown is evident in the number of prescriptions and number of DDD/1000 patients for psychiatric and cardiovascular diseases but less for diabetes.

As shown in table 2, the number of patients with cardiovascular diseases slightly decreased. Patients with diabetes showed a small increase. Patients with psychiatric disorders were greatly diminished.

Some differences were seen among different ethnic groups. The small group of indigent Italians showed no reduction in the number of patients with chronic conditions attending OSF, including those with psychiatric problems. On the contrary, they showed a general increase.

Female patients receiving medications for chronic conditions showed a greater reduction than males.

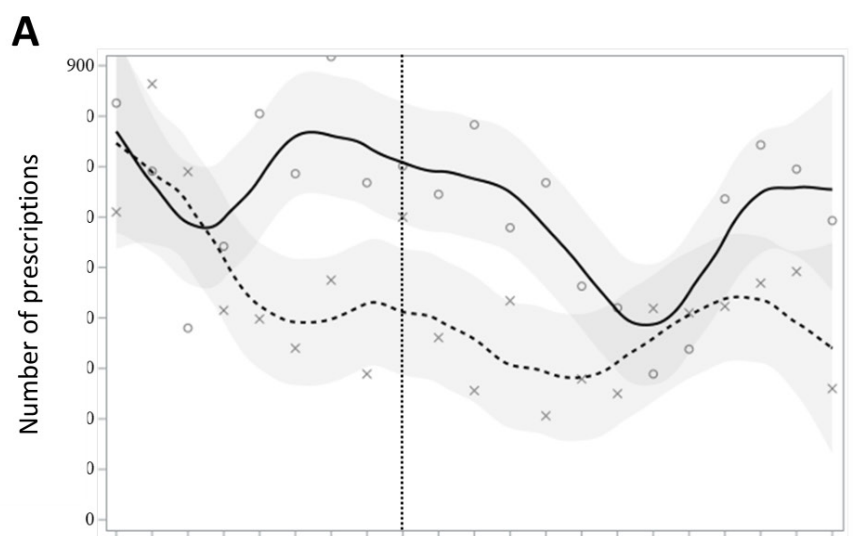

B

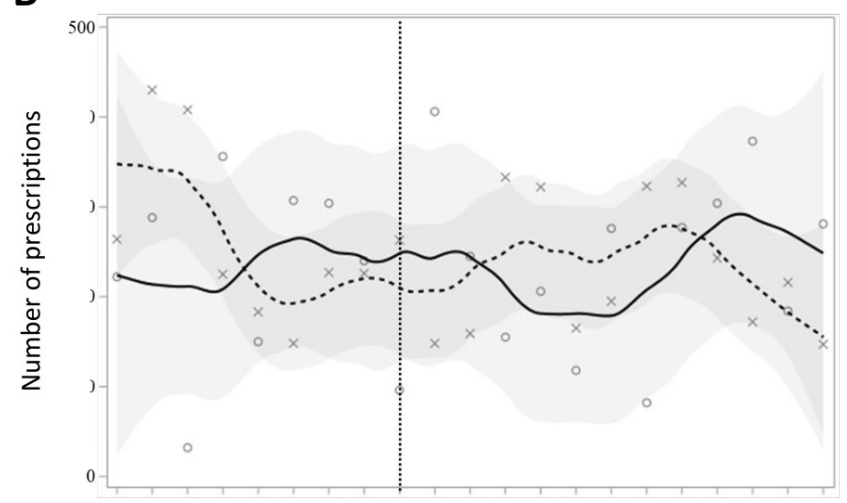

C

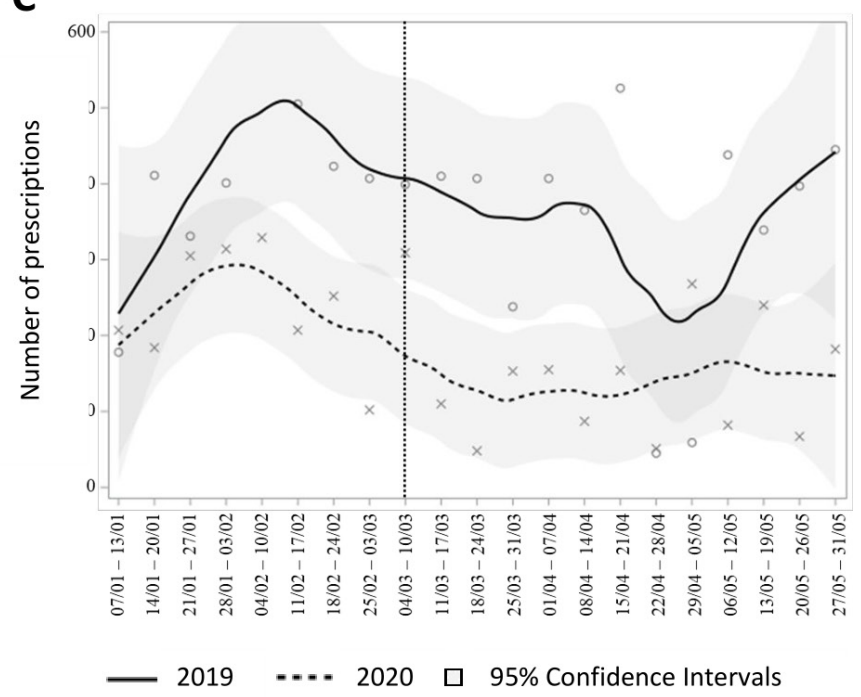

Figure 2 The figure shows the trend of prescriptions of cardiovascular (A), antidiabetic (B) and psychiatric (C) drugs by OSF during the period from 7 January 2019 to 31 May 2019 (solid line) and in the same period of 2020 (dotted line). The vertical line indicates the date the lockdown was imposed in Italy (ie,8 March 2020). Trends of the number of prescriptions were smoothed using the LOESS method, in order to remove seasonality and casual fluctuations over time. Circles and crosses represent the number of prescriptions observed in 2019 and in 2020, respectively. The grey areas represent $95 \%$ Cls. OSF.

\section{DISCUSSION}

This study describes the effects of SARS-CoV-2 pandemic and the consequent lockdown on a population of 

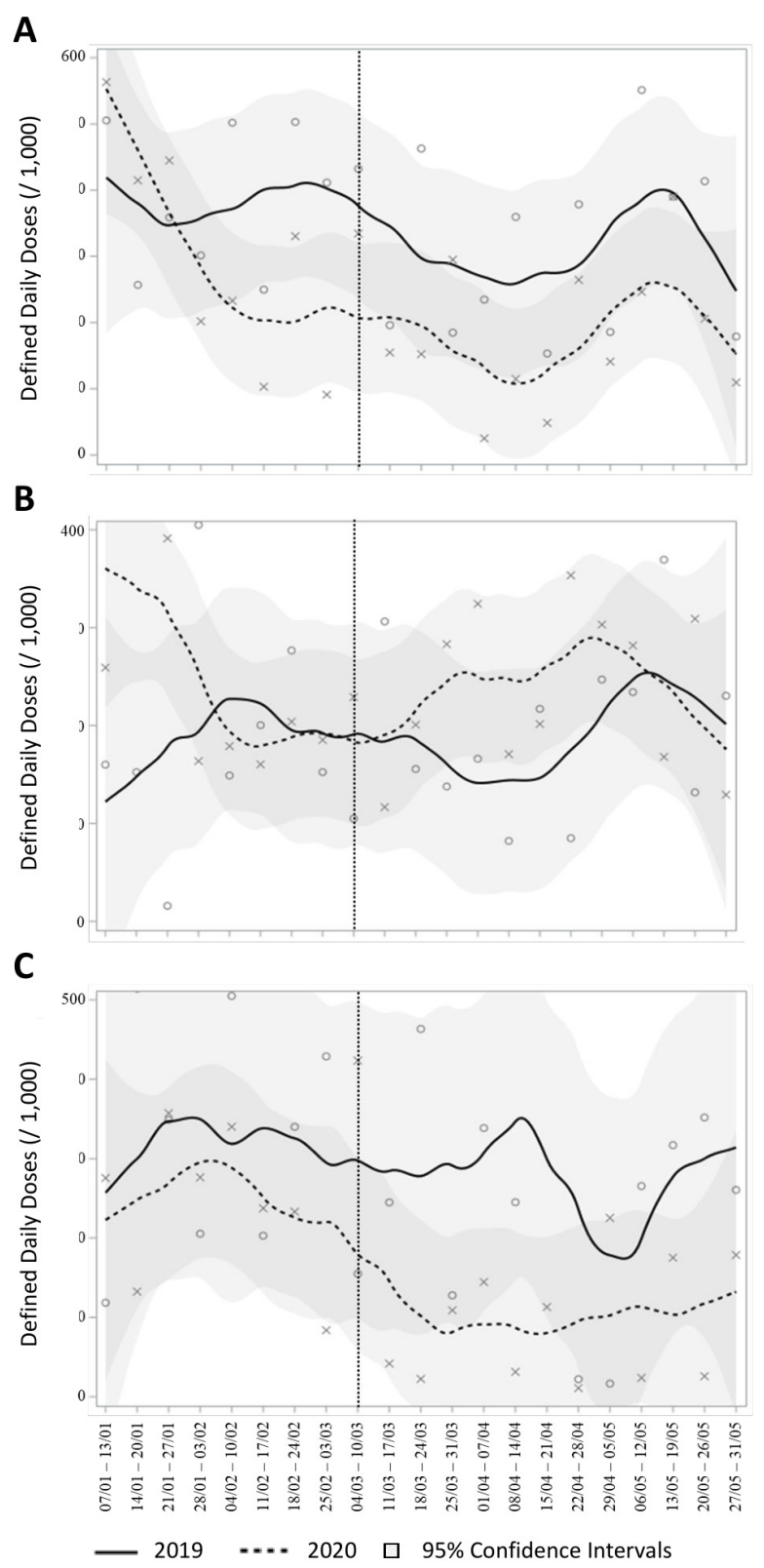

Figure 3 The figure shows the dispensed quantity (measured as total DDD/1000 patients) of cardiovascular (A), antidiabetic (B) and psychiatric (C) drugs during the period from 7 January 2019 to 31 May 2019 (solid line) and the same period in 2020 (dotted line). The vertical line indicates the date the lockdown was imposed in Italy (ie,8 March 2020). Trends of dispensed quantities were smoothed using the LOESS method, in order to remove seasonality and casual fluctuations over time. Circles and crosses represent the quantities observed in 2019 and in 2020, respectively. The grey areas represent $95 \%$ Cls.

undocumented migrants receiving medical assistance from an NGO in Milan, Italy. It has to be noticed that, unlike other NGO operating in the same area, OSF continued its activity regularly during the lockdown with not even 1 day of suspension. Therefore, our observations were not biased by periods of closure and shortages in medications supply.
However, we are aware that our study has some limitations.

First, we could not predetermine the magnitude of our sample, but we simply used all available data. Second, we cannot say which percentage our patients represent of all undocumented migrants living in Milan. Third, we had no means of contacting patients lost at follow-up; therefore, the reasons for this could only be inferred.

Even with these limitations, we try to make some considerations.

Our data show a generalised reduction in the number of patients requiring to be treated. The number of prescriptions was also reduced, as was the quantity (measured as DDD/1000 patients) of dispensed medications.

This was true also for patients with NCD, though it is known that undocumented migrants are frequently affected by these diseases. ${ }^{27}$

An exception appears to be represented by patients with diabetes, who did not show significant reductions. Indeed, their number and their need of pharmacological treatment increased during the lockdown period as compared with the first months of 2020. Though this can be due to the different length of the two periods, it should be considered that this did not happened with other NCD. Incidence and severity of COVID-19 could possibly be increased in patients with diabetes. ${ }^{28}{ }^{29}$ Awareness of this potential risk could have led diabetic undocumented migrants to a better adherence to therapy and follow-up. This interpretation is in keeping with our previous observation that undocumented migrants tend to be highly aware of their chronic diseases. ${ }^{30}$

In a general context, it has been noticed that COVID-19 pandemic had both negative ${ }^{31}$ and positive ${ }^{32}$ effects on lifestyle and consequently on glycaemic control of patients with diabetes. Positive effects could have been predominant in the population of our study, though we have no explanation for this.

Things went much worse with other NCD, especially psychiatric disorders. This is quite surprising since we know that these problems are not uncommon in undocumented migrants and more in general in the population cared for by OSF. ${ }^{33}$

Moreover, COVID-19 pandemic had multifaceted psychological implications, including an increased incidence of depressive symptoms. ${ }^{73435}$ Therefore, we would have expected a greater demand for psychiatric consultations and medicines during the period of quarantine. This has not been the case.

Since it is known that psychological disorders, also during the pandemic, were at least in part dependent from modifiable factors, ${ }^{78}$ it is possible that some of these, as support from family and friends, had a role, at least in some groups of patients. Exposure to social media has also been shown to be a significant worsening factor, especially for anxiety. ${ }^{36} \mathrm{~A}$ part of our patients is homeless, while the vast majority live in overcrowded households. In both cases, the intensive use of electronic devices is impossible. Moreover, the vast majority of them has no 
Table 1 Number of patients prescribed a medication for cardiovascular diseases, diabetes and psychiatric disorders in the lockdown period (8 March 2020 to 31 May 2020) and in the same period of 2019, stratified by sex, age and ethnicity

\begin{tabular}{|c|c|c|c|c|c|c|c|c|c|}
\hline & \multicolumn{3}{|c|}{ Cardiovascular } & \multicolumn{3}{|c|}{ Diabetes } & \multicolumn{3}{|c|}{ Psychiatric } \\
\hline & 2019 & 2020 & $\%$ variation & 2019 & 2020 & $\%$ variation & 2019 & 2020 & $\%$ variation \\
\hline \multicolumn{10}{|l|}{ Age (years) } \\
\hline $0-17$ & 1 & 0 & -100 & 0 & 1 & & 0 & 0 & 0 \\
\hline $18-64$ & 349 & 254 & -27 & 126 & 114 & -10 & 217 & 145 & -33 \\
\hline$\geq 65$ & 96 & 68 & -29 & 27 & 23 & -15 & 32 & 11 & -66 \\
\hline \multicolumn{10}{|l|}{ Sex } \\
\hline Female & 243 & 159 & -35 & 73 & 63 & -14 & 148 & 85 & -43 \\
\hline Male & 203 & 163 & -20 & 80 & 75 & -6 & 101 & 71 & -30 \\
\hline \multicolumn{10}{|l|}{ Ethnicity } \\
\hline Mediterranean Africa & 42 & 35 & -17 & 18 & 19 & 6 & 19 & 13 & -32 \\
\hline Sub-Saharan Africa & 35 & 17 & -51 & 10 & 5 & -50 & 28 & 18 & -36 \\
\hline Latin America & 167 & 116 & -31 & 63 & 48 & -24 & 98 & 61 & -38 \\
\hline Asia & 40 & 35 & -13 & 19 & 22 & 16 & 19 & 17 & -11 \\
\hline Eastern Europe & 145 & 101 & -30 & 39 & 39 & 0 & 55 & 26 & -53 \\
\hline Italy & 13 & 14 & 12 & 2 & 3 & 50 & 26 & 18 & -35 \\
\hline Other/missing & 4 & 4 & 0 & 2 & 2 & 0 & 4 & 3 & -25 \\
\hline Total & 446 & 322 & -28 & 153 & 138 & -10 & 249 & 156 & -37 \\
\hline
\end{tabular}

hardware enabling them to stay connected for long periods. The only exception is the mobile phone, but also this is very frequently outdated. Therefore, patients attending OSF were undoubtedly less exposed to social media. This might have contributed to reduce their anxiety and their need for anxiolytic medications.
Patients with cardiovascular problems experienced a minor reduction in the lockdown period as compared with the prelockdown months of 2020, though they showed a great reduction between the same months of 2019 and 2020. The latter can be an expression of the great variability of this unstable population. The former is

Table 2 Number of patients prescribed a medication for cardiovascular diseases, diabetes and psychiatric disorders in the lockdown period (8 March 2020 to 31 May 2020) and in the prelockdown period (7 January 2020 to 7 March 2020), stratified by sex, age and ethnicity

\begin{tabular}{|c|c|c|c|c|c|c|c|c|c|}
\hline & \multicolumn{3}{|c|}{ Cardiovascular } & \multicolumn{3}{|c|}{ Antidiabetic } & \multicolumn{3}{|c|}{ Psychiatric } \\
\hline & Prelockdown & Lockdown & $\begin{array}{l}\% \\
\text { variation }\end{array}$ & Prelockdown & Lockdown & $\%$ variation & Prelockdown & Lockdown & $\begin{array}{l}\% \\
\text { variation }\end{array}$ \\
\hline \multicolumn{10}{|l|}{ Age (years) } \\
\hline $0-17$ & 0 & 0 & 0 & 2 & 1 & -50 & 0 & 0 & 0 \\
\hline $18-64$ & 245 & 254 & 4 & 89 & 114 & 28 & 186 & 145 & -22 \\
\hline$\geq 65$ & 86 & 68 & -21 & 31 & 23 & -26 & 20 & 11 & -45 \\
\hline \multicolumn{10}{|l|}{ Sex } \\
\hline Female & 175 & 159 & -9 & 57 & 63 & 11 & 129 & 85 & -34 \\
\hline Male & 156 & 163 & 4 & 65 & 75 & 15 & 77 & 71 & -8 \\
\hline \multicolumn{10}{|l|}{ Ethnicity } \\
\hline $\begin{array}{l}\text { Mediterranean } \\
\text { Africa }\end{array}$ & 24 & 35 & 46 & 16 & 19 & 19 & 16 & 13 & -19 \\
\hline $\begin{array}{l}\text { Sub-Saharan } \\
\text { Africa }\end{array}$ & 14 & 17 & 21 & 6 & 5 & -17 & 18 & 18 & 0 \\
\hline Latin America & 132 & 116 & -12 & 53 & 48 & -9 & 88 & 61 & -31 \\
\hline Asia & 35 & 35 & 0 & 11 & 22 & 100 & 14 & 17 & 21 \\
\hline Eastern Europe & 107 & 101 & -6 & 32 & 39 & 22 & 49 & 26 & -47 \\
\hline Italy & 11 & 14 & 27 & 2 & 3 & 50 & 17 & 18 & 6 \\
\hline Other/missing & 8 & 4 & -50 & 2 & 2 & 0 & 4 & 3 & -25 \\
\hline Total & 331 & 322 & -3 & 122 & 138 & 13 & 206 & 156 & -24 \\
\hline
\end{tabular}


more difficult to explain but is in line with the reduction in cardiovascular diagnoses in the general population during the same period. ${ }^{18}{ }^{37}$ Moreover, a part of patients in the cardiovascular group were patients with arterial hypertension. Therefore, it is possible that, in the absence of symptoms, some of them have suspended treatments and consultations.

Though we know that during COVID-19 pandemic all patients with NCD experienced reduced medical assistance, ${ }^{16} 38$ we do not know exactly why undocumented migrants reduced consultations and requests of medicines during the lockdown. The considerations made previously could explain this trend which, with the exception of diabetes, has been generalised, but we cannot be sure that they are the only ones. Moreover, it is interesting to note that indigent Italians were less involved. Though they were quite few, we noticed that their number increased during lockdown, with the only exception of patient with psychiatric disorders, who were less than in the same months of 2019. This is interesting since we observed that, among the poor, awareness of diseases is not higher in natives than in migrants. ${ }^{30} \mathrm{~A}$ different explanation has to be looked for. However, the number of indigent Italians in the present study was so small that it does not allow to draw any conclusion.

We must take into account that undocumented migrants might have been kept away from medical assistance for different reasons. In general, disadvantaged segments of population face more difficulties in accessing usual forms of care. ${ }^{39} 40$ In the case of undocumented migrants, these barriers are usually related to law and policy, but this is surely not the case with OSF. However, recently arrived immigrants, not knowing the real situation, could have been discouraged by this sort of feeling. More likely, the need to break containment rules to access the outpatient clinic could have acted as a deterrent, since being identified as undocumented puts a person at risk of being expelled. This can also explain the different behaviour of undocumented migrants and natives. On the contrary, it does not explain why undocumented migrants with diabetes continued to access the outpatient clinics of OSF, while those with other NCDs showed a great reduction.

Another interesting fact is that the decrease of the number of female patients accessing OSF outpatient clinics was greater than that of males. This is another observation that is difficult to explain, especially considering that females could be more susceptible than males to some health problems as, for example, psychological disorders. ${ }^{41}{ }^{42}$ Though it has been suggested that gender discrimination can increase during lockdown, ${ }^{43}$ this seems hardly the only explanation of the difference we found between female and male patients.

Taken together, our findings seem to suggest that a greater involvement of health authorities in taking care of the more indigent segments of our population is necessary.

To work in this direction, a good knowledge of the health needs of these persons is an inevitable first step.
The method we describe in this study can be useful, but it is far from being exhaustive. Moreover, it can be used only with relatively small groups of patients, such as those attending charitable facilities.

Further investigation should evaluate other methods of getting the information we need.

One could be the use of questionnaires. Of course, mail questionnaires are impossible with this type of patients, and also telephone interviews show many difficulties. On the contrary, questionnaires administered directly on site, before or after consultations, seem to be a promising tool.

They could be administered to different groups of patients attending different charities and so it could be possible to get more comprehensive information on their needs. This seems worth to be investigated.

Telemedicine is probably an even more promising tool. During the COVID-19 outbreak, it has been successfully used, for example, with neurological patients. ${ }^{44}$ This system has been demonstrated to be accessible and reliable. ${ }^{45}$ In its basic form, it can at least transmit basic life parameters, which is essential to escalate the level of care. ${ }^{46}$ Therefore, for patients like those described in this study, it could be used to get information and to send instructions or even to locate patients needing emergency interventions. It is true that with so unstable and indigent persons, we can expect many difficulties in its implementation. However, it seems to be a valuable and affordable strategy, ${ }^{47}$ and it is definitely worth to be further investigated. As mentioned previously, these persons usually have a mobile phone, so the first subject of research could be its use to implement some form of telemedicine.

This type of studies can really help to know more accurately the health needs of these patients and to offer them a better assistance, while we wait for a more inclusive assistance for all the persons who need it, in spite of their administrative status.

\section{CONCLUSION}

In the present study, we show that undocumented migrants living in a Western country changed their way to use healthcare facilities offered by an NGO during the lockdown period. This change was uneven. Patients with different chronic conditions behave differently: a significant decrease in the number of consultations and medicine dispensations was seen for patients with mental disturbances, while no relevant change was noticed for patients with diabetes. Females suffered a greater reduction than males.

It is also possible that undocumented migrants and natives in the same conditions of poverty had to face different barriers to access free healthcare services.

Now we feel that recovery is at hand, and authoritative speakers have underlined the need for it to be an equitable one at a global level. ${ }^{48}$ Which will be the consequences if it is not equitable even at a local level? 
Author affiliations

${ }^{1}$ Laboratory of Clinical Pharmacology and Pharmacoepidemiology, Department of Clinical Sciences and Community Health, University of Milan, Milan, Italy

${ }^{2}$ National Centre for Healthcare Research and Pharmacoepidemiology, Milan, Italy ${ }^{3}$ Laboratory of Healthcare Research and Pharmacoepidemiology, Department of Statistics and Quantitative Methods, University of Milano Bicocca, Milan, Italy ${ }^{4}$ Opera San Francesco per i Poveri, Milan, Italy ${ }^{5}$ Istituto Auxologico Italiano, IRCCS, Experimental Laboratory for AuxoEndocrinological Research, 28824 Verbania, Italy

Acknowledgements The help of Laura Lia with the electronic data sets is gratefully acknowledged.

Contributors GF and SGC designed the study and wrote the manuscript; MF and RT performed the statistical analysis; SF and AER collected the data; GC and AS contributed to the interpretation of the results.

Funding This work was supported by grants from the Italian Ministry of Education, University and Research ('PRIN' 2017, project 2017728JPK).

Competing interests None declared.

Patient consent for publication Not required.

Ethics approval This study was approved by the Ethics Committee of the University of Milano Bicocca. Protocol title: Prestazioni sanitarie erogate da una Organizzazione non Governativa a migranti irregolari e Italiani poveri durante il primo periodo di lockdown per Covid-19COVID-19. Confronto con lo stesso periodo dell'anno precedente e col periodo pre-lockdown. Approval No. 574, 11 December 2020 . All data were completely and permanently anonymised. All procedures were in accordance with the ethical standards of the institutional and national research committee and with the 1964 Helsinki declaration and its later amendments or comparable ethical standards.

Provenance and peer review Not commissioned; externally peer reviewed by Elaine Macaninch, Brighton and Sussex Medical School and University Hospitals Sussex, UK

Data availability statement The data used to support the findings of this study are available from the corresponding author on reasonable request.

Open access This is an open access article distributed in accordance with the Creative Commons Attribution Non Commercial (CC BY-NC 4.0) license, which permits others to distribute, remix, adapt, build upon this work non-commercially, and license their derivative works on different terms, provided the original work is properly cited, appropriate credit is given, any changes made indicated, and the use is non-commercial. See: http://creativecommons.org/licenses/by-nc/4.0/.

\section{REFERENCES}

1 Prezioso C, Pietropaolo V. COVID-19: update of the Italian situation. J Neurovirol 2020;26:834-7.

2 Jayaweera M, Perera H, Gunawardana B, et al. Transmission of COVID-19 virus by droplets and aerosols: a critical review on the unresolved dichotomy. Environ Res 2020;188:109819.

3 Lau H, Khosrawipour V, Kocbach P, et al. The positive impact of lockdown in Wuhan on containing the COVID-19 outbreak in China. $J$ Travel Med 2020;27:1-7.

4 Mills EHA, Møller AL, Gnesin F, et al. National all-cause mortality during the COVID-19 pandemic: a Danish registry-based study. Eur J Epidemiol 2020;35:1007-19.

5 Silverio A, Di Maio M, Ciccarelli M, et al. Timing of national lockdown and mortality in COVID-19: the Italian experience. Int $J$ Infect Dis 2020;100:193-5.

6 Brooks SK, Webster RK, Smith LE, et al. The psychological impact of quarantine and how to reduce it: rapid review of the evidence. Lancet 2020;395:912-20.

7 Gualano MR, Lo Moro G, Voglino G, et al. Effects of Covid-19 lockdown on mental health and sleep disturbances in Italy. Int J Environ Res Public Health 2020;17. doi:10.3390/ijerph17134779. [Epub ahead of print: 02 Jul 2020].

8 Killgore WDS, Taylor EC, Cloonan SA, et al. Psychological resilience during the COVID-19 lockdown. Psychiatry Res 2020;291:113216.

9 Guessoum SB, Lachal J, Radjack R, et al. Adolescent psychiatric disorders during the COVID-19 pandemic and lockdown. Psychiatry Res 2020;291:113264.

10 Yuki K, Fujiogi M, Koutsogiannaki S. COVID-19 pathophysiology: a review. Clin Immunol 2020;215:108427.
11 Davies NG, Klepac P, Liu Y, et al. Age-dependent effects in the transmission and control of COVID-19 epidemics. Nat Med 2020;26:1205-11.

12 Musanabaganwa C, Cubaka V, Mpabuka E, et al. One hundred thirtythree observed COVID-19 deaths in 10 months: unpacking lower than predicted mortality in Rwanda. BMJ Glob Health 2021;6.

13 Flaherty GT, Hession P, Liew CH, et al. COVID-19 in adult patients with pre-existing chronic cardiac, respiratory and metabolic disease: a critical literature review with clinical recommendations. Trop Dis Travel Med Vaccines 2020;6:16.

14 Williams J, Allen L, Wickramasinghe K, et al. A systematic review of associations between non-communicable diseases and socioeconomic status within low- and lower-middle-income countries. J Glob Health 2018;8:020409.

15 Palmer K, Monaco A, Kivipelto M, et al. The potential longterm impact of the COVID-19 outbreak on patients with noncommunicable diseases in Europe: consequences for healthy ageing. Aging Clin Exp Res 2020;32:1189-94.

16 Saqib MAN, Siddiqui S, Qasim M, et al. Effect of COVID-19 lockdown on patients with chronic diseases. Diabetes Metab Syndr 2020;14:1621-3.

17 Chan DZL, Stewart RAH, Kerr AJ. The impact of a national COVID-19 lockdown on acute coronary syndrome hospitalisations in New Zealand (ANZACS-QI 55). Lancet Regional Health 2020;5:100056.

18 Mesnier J, Cottin Y, Coste P, et al. Hospital admissions for acute myocardial infarction before and after lockdown according to regional prevalence of COVID-19 and patient profile in France: a registry study. Lancet Public Health 2020;5:e536-42.

19 Sydenstricker $\mathrm{E}$. The incidence of influenza among persons of different economic status during the epidemic of 1918.. Public Health Rep 2006;121 Suppl 1:191-204; discussion 190.

20 Haider N, Osman AY, Gadzekpo A, et al. Lockdown measures in response to COVID-19 in nine sub-Saharan African countries. BMJ Glob Health 2020;5:e003319.

21 Mikolai J, Keenan K, Kulu H. Intersecting household-level health and socio-economic vulnerabilities and the COVID-19 crisis: an analysis from the UK. SSM Popul Health 2020;12:100628.

22 Mukumbang FC, Ambe AN, Adebiyi BO. Unspoken inequality: how COVID-19 has exacerbated existing vulnerabilities of asylumseekers, refugees, and undocumented migrants in South Africa. Int $J$ Equity Health 2020;19:141.

23 Dyer O. Covid-19: black people and other minorities are hardest hit in US. BMJ 2020;369:m1483.

24 Methodology WCC for DS. Guidelines for ATC classification and DDD assignment, 2020; WHO collaborating centre for drug statistics methodology. Oslo, Norway, 2019.

25 Fiorini G, Cortinovis I, Corrao G, et al. Current pharmacological treatment of type 2 diabetes mellitus in undocumented migrants: is it appropriate for the phenotype of the disease? Int J Environ Res Public Health 2020;17:8169.

26 Cleveland WS. Robust locally weighted regression and smoothing Scatterplots. J Am Stat Assoc 1979;74:829-36.

27 Fiorini G, Cerri C, Bini S, et al. The burden of chronic noncommunicable diseases in undocumented migrants: a 1-year survey of drugs dispensation by a non-governmental organization in Italy. Public Health 2016;141:26-31.

28 Singh AK, Gupta R, Ghosh A, et al. Diabetes in COVID-19: prevalence, pathophysiology, prognosis and practical considerations. Diabetes Metab Syndr 2020;14:303-10.

29 Tadic M, Cuspidi C, Sala C. COVID-19 and diabetes: is there enough evidence? J Clin Hypertens 2020;22:943-8.

30 Fiorini G, Cerri C, Magri F, et al. Risk factors, awareness of disease and use of medications in a deprived population: differences between indigent natives and undocumented migrants in Italy. $J$ Public Health 2021;43:302-7.

31 Ruiz-Roso MB, Knott-Torcal C, Matilla-Escalante DC, et al. COVID-19 lockdown and changes of the dietary pattern and physical activity habits in a cohort of patients with type 2 diabetes mellitus. Nutrients 2020;12:2327.

32 Nachimuthu S, Vijayalakshmi R, Sudha M, et al. Coping with diabetes during the COVID - 19 lockdown in India: Results of an online pilot survey. Diabetes Metab Syndr 2020;14:579-82.

33 Cerri C, Fiorini G, Bini S, et al. Psychotropic drugs prescription in undocumented migrants and indigent natives in Italy. Int Clin Psychopharmacol 2017;32:294-7.

34 Bonati M, Campi R, Zanetti M, et al. Psychological distress among Italians during the 2019 coronavirus disease (COVID-19) quarantine. BMC Psychiatry 2021;21:20.

35 Volken T, Zysset A, Amendola S, et al. Depressive symptoms in Swiss university students during the COVID-19 pandemic and its correlates. Int J Environ Res Public Health 2021;18:1458. 
36 Gao J, Zheng P, Jia Y, et al. Mental health problems and social media exposure during COVID-19 outbreak. PLoS One 2020;15:e0231924.

37 Holt A, Gislason GH, Schou M, et al. New-onset atrial fibrillation: incidence, characteristics, and related events following a national COVID-19 lockdown of 5.6 million people. Eur Heart J 2020;41:3072-9.

38 Mauro V, Lorenzo M, Paolo C, et al. Treat all COVID 19-positive patients, but do not forget those negative with chronic diseases. Intern Emerg Med 2020;15:787-90.

39 Hacker K, Anies M, Folb BL, et al. Barriers to health care for undocumented immigrants: a literature review. Risk Manag Healthc Policy 2015;8:175-83.

40 Elwell-Sutton T, Fok J, Albanese F, et al. Factors associated with access to care and healthcare utilization in the homeless population of England. J Public Health 2017;39:26-33.

41 Costantini A, Mazzotti E. Italian validation of COVID-19 Peritraumatic distress index and preliminary data in a sample of general population. Riv Psichiatr 2020;55:145-51.

42 Tzur Bitan D, Grossman-Giron A, Bloch Y, et al. Fear of COVID-19 scale: psychometric characteristics, reliability and validity in the Israeli population. Psychiatry Res 2020;289:113100.
43 Shammi M, Bodrud-Doza M, Islam ARMT, et al. Strategic assessment of COVID-19 pandemic in Bangladesh: comparative lockdown scenario analysis, public perception, and management for sustainability. Environ Dev Sustain 2020;18:1-44.

44 Furlanis G, Ajčević M, Naccarato M, et al. E-Health vs COVID-19: home patient telemonitoring to maintain TIA continuum of care. Neurol Sci 2020;41:2023-4.

45 Blasioli E, Hassini E. E-Health technological ecosystems: advanced solutions to support informal caregivers and vulnerable populations during the COVID-19 outbreak. Telemed J E Health 2021. doi:10.1089/tmj.2020.0522. [Epub ahead of print: $21 \mathrm{Apr}$ 2021]

46 Ko SQ, Hooi BMY, Koo C-Y, et al. Remote monitoring of marginalised populations affected by COVID-19: a retrospective review. BMJ Open 2020;10:e042647.

47 Wang LY, Low TT, Yeo TJ. Telehealth in COVID-19 and cardiovascular disease-Ensuring equitable care. Ann Acad Med Singap 2020;49:902-4.

48 Loewenson R, D'Ambruoso L, Duc DM, et al. Equitable recovery from COVID-19: bring global commitments to community level. BMJ Glob Health 2021;6:e004757. 